Corrigendum

\title{
The discordant method: a novel approach for differential correlation
}

\section{Charlotte Siska, Russell Bowler and Katerina Kechris}

Bioinformatics (2016) 32(5), 690-696. doi:10.1093/bioinformatics/ btv633

The authors of the above paper wish to inform readers that there was a typographical error in Equations 2 and 6 of the published paper. The corrected equations are given below. The paper has now been corrected online.

Equation 2:

$$
f\left[z^{1}, z^{2}\right]=\prod_{i=0}^{2} \prod_{j=0}^{2}\left(\pi_{i j} \phi_{\mu_{i}, \sigma_{i}^{2}}\left[z^{1}\right] \phi_{\eta_{j}, \tau_{j}^{2}}\left[z^{2}\right]\right)^{1\left(w_{i j}=1\right)}
$$

Equation 6:

$$
z^{*}=\frac{z_{2}-z_{1}}{\sqrt{\frac{1}{n_{2}-3}-\frac{1}{n_{1}-3}}}
$$

